09

\title{
Влияние поляризационных характеристик пробного излучения на сигнал оптически детектируемого магнитного резонанса в магнитометрических и гироскопических квантовых датчиках
}

\author{
(C) А.К. Вершовский, С.П. Дмитриев, А.С. Пазгалев, М.В. Петренко \\ Физико-технический институт им. А.Ф. Иоффре РАН, Санкт-Петербург, Россия \\ E-mail: antver@mail.ioffe.ru
}

Поступило в Редакцию 14 июня 2019 г.

В окончательной редакции 14 июня 2019 г.

Принято к публикации 25 июня 2019 г.

\begin{abstract}
Рассматривается влияние поляризационных характеристик пробного лазерного излучения в схемах квантовых датчиков (магнитометров на основе эффекта электронного парамагнитного резонанса и гироскопов, использующих одновременно эффекты электронного и ядерного магнитного резонанса) на амплитуду сигнала этих датчиков. Выведены и экспериментально проверены использующие формализм Стокса-Мюллера соотношения, связывающие величину сигнала магнитного резонанса с параметрами элементов оптической схемы датчика. Показано, что основное деструктивное влияние на сигнал в стандартной двухлучевой схеме оказывают фазовые задержки, вносимые как металлическими, так и диэлектрическими зеркалами. Предложены и апробированы методы компенсации этого деструктивного влияния.
\end{abstract}

Ключевые слова: оптически детектируемый магнитный резонанс, квантовый магнитометр, поляризация света.

DOI: 10.21883/PJTF.2019.20.48383.17930

Два последних десятилетия характеризуются возрождением и бурным ростом интереса к квантовым датчикам, использующим оптическую накачку и оптическое детектирование магнитного резонанса в ячейках, содержащих пары щелочных атомов $[1,2]$. Этот интерес обусловлен в первую очередь появлением надежных компактных источников резонансного лазерного излучения, которое в этих датчиках используется как для накачки, так и для детектирования резонанса. В частности, с появлением лазеров с вертикально-излучающим резонатором $[3,4]$ стала возможной разработка сверхкомпактных магнитометров [5,6] и гироскопов на основе эффекта ядерного магнитного резонанса [7,8]. Эти схемы получили дальнейшее развитие благодаря использованию таких нелинейных эффектов, как сужение линии магнитного резонанса при высокой интенсивности лазерной накачки [9] и в нулевых магнитных полях [10,11]. В результате чувствительность оптических магнитометрических датчиков была повышена настолько, что позволила им конкурировать со сверхпроводящими датчиками в магнитоэнцефалографических системах [12].

В классической двухлучевой схеме датчика [13] оптическая накачка атомов щелочного металла осуществляется циркулярно поляризованным лучом, настроенным на центр линии поглощения и направленным вдоль оси $z$, т.е. вдоль магнитного поля, а для детектирования используется пробный луч, направленный вдоль оси $x$ (рис. 1). Максимальная чувствительность, а также степень подавления флуктуаций лазерного излучения реализуются при детектировании пробным линейно поляризованным лучом, отстроенным по частоте от цен- тра линии оптического поглощения щелочного металла на несколько ее ширин. Магнитные моменты атомов в постоянном поле прецессируют на так называемой ларморовской частоте в плоскости $x 0 y$; резонансное радиочастотное поле выравнивает фазы прецессий отдельных моментов. Поперечная компонента коллективного момента создает в системе осциллирующее циркулярное двулучепреломление, вследствие чего азимут плоскости поляризации пробного луча также осциллирует на частоте магнитного резонанса. Эти осцилляции детектируются измерителем угла поляризации, в простейшем случае представляющим собой балансный фотоприемник (БФП), состоящий из поляризационного расщепителя и двух детекторов интенсивности излучения.

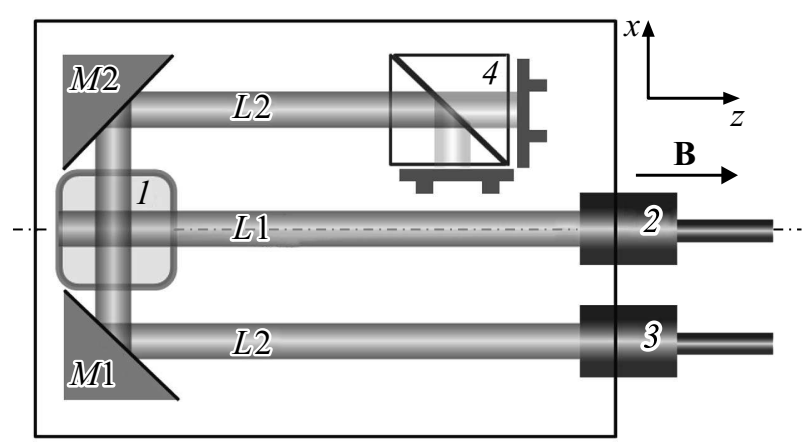

Рис. 1. Упрощенная схема квантового датчика. 1 - ячейка, 2 - ввод излучения накачки, 3 - ввод пробного излучения, 4 - балансный фотоприемник, $L 1-$ луч оптической накачки, $L 2$ - пробный луч, $M 1, M 2$ - зеркала. 
Параметры зеркал

\begin{tabular}{c|c|c|c|c}
\hline Тип & Обозначение & $R_{p}$ & $R_{s}$ & $\chi,^{\circ}$ \\
\hline Алюминиевое & $\mathrm{Al}$ & $0.876^{*}$ & 0.936 & 4.911 \\
Алюминиевое (эксперимент) & $\mathrm{Al}(\operatorname{Exp})$ & $0.848^{*}$ & $0.936^{*}$ & $7.9^{*}$ \\
Алюминиевое с покрытием & $\mathrm{Al}+\mathrm{SiO}_{2}$ & $0.848^{*}$ & $0.942^{*}$ & $17.0^{*}$ \\
Серебряное & $\mathrm{Ag}$ & 0.979 & 0.989 & 9.424 \\
Золотое & $\mathrm{Au}$ & 0.940 & 0.969 & 1.492 \\
Диэлектрическое & Dielectr. & $0.990^{* *}$ & $0.995^{* *}$ & $38.0^{*}$
\end{tabular}

* Данные получены в эксперименте.

** Данные взяты из каталога производителя.

Требования компактности заставляют в большинстве случаев использовать на практике схему, в которой детектирующий луч входит в датчик параллельно лучу накачки, а на входе в ячейку и на выходе из нее поворачивается на $90^{\circ}$ зеркалами $M 1$ и $M 2$. Эти зеркала неизбежно влияют на поляризационные характеристики пробного луча $[14,15]$, снижая эффективность детектирования сигнала магнитного резонанса. Настоящая работа посвящена исследованию этого влияния и разработке методов его минимизации. Параметры магнитного резонанса в ячейке считаем не зависящими от параметров пробного луча; при условии нормировки интенсивности пробного света на БФП от них также не зависит среднеквадратичная амплитуда дробовых шумов, и фактор качества магнитного резонанса $[2,16]$ определяется амплитудой сигнала, регистрируемого фотоприемником.

Магнитный резонанс возбуждался в ячейке размером $8 \times 8 \times 8 \mathrm{~mm}$, содержащей насыщенные пары Cs и азот $\left(\mathrm{N}_{2}\right)$ под давлением 100 Torr; в качестве источников накачки и пробного излучения использовались полупроводниковые лазеры с внешними резонаторами производства компании VitaWave. Ячейка была расположена в многослойном магнитном экране, индукция поля в котором поддерживалась на уровне $12.0 \mu \mathrm{T}$. БФП представлял собой кубический поляризационный расщепитель Thorlabs PBS122 с двумя фотодиодами в общей вращающейся оправе. Исследование поляризационных характеристик пробного луча производилось с помощью поляриметра Thorlabs PAX5710IR1-T-TXP. Амплитуда сигнала на ларморовской частоте измерялась синхронным детектором SR830.

Для описания характеристик пробного луча, оптических элементов схемы и рабочей ячейки нами использовался формализм вектора Стокса [17] в случае полностью поляризованного света, характеризуемого тремя независимыми параметрами: интенсивностью $I$, азимутом поляризации $\psi$ и эллиптичностью $\chi$. Все оптические элементы описывались комбинациями элементарных матриц Мюллера [18]: $R(\theta)$ - оператор вращения на угол $\theta ; D(\delta)$ - оператор фазовой задержки на угол $\delta ; A\left(R_{p}, R_{s}\right)$ - оператор потерь на поверхностях, $R_{p}, R_{s}$ - коэффициенты отражения для $p$ - и $s$ поляризации соответственно. Зеркало в таком формализме описывается матрицей $M\left(R_{p}, R_{s}, \delta\right)=A\left(R_{p}, R_{s}\right) D(\delta)$
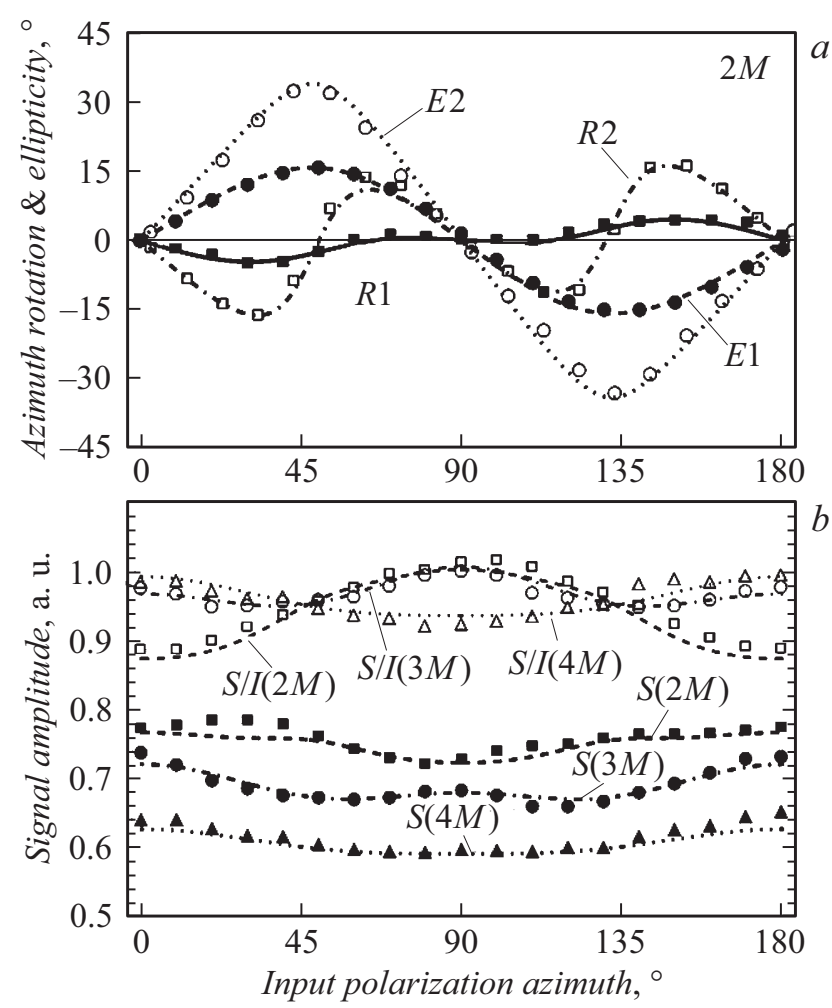

Рис. 2. $a-$ зависимость от азимута входной поляризации поворота азимута выходной поляризации $(R 1, R 2)$ и эллиптичности $(E 1, E 2)$ для систем из двух зеркал - $\mathrm{Al}(1)$ и $\mathrm{Al}+\mathrm{SiO}_{2}(2) ; b-$ амплитуды сигнала $(S)$ и амплитуды сигнала, нормированной на интенсивность $(S / I)$, от азимута входной поляризации для схем с двумя $(2 M)$, тремя $(3 M)$ и четырьмя (4M) Al-зеркалами. Точки - эксперимент, линии теория.

(где $\delta=2 \chi_{M}$, а $\chi_{M}-$ максимальная эллиптичность, вносимая зеркалом), произвольная фазовая пластина, оси которой наклонены под углом $\theta$ к вертикали, матрицей $P(\theta, \delta)=R(\theta) D(\delta) R(-\theta)$. Было рассмотрено несколько типов зеркал, параметры которых [19] приведены в таблице.

Отметим, что измеренные нами параметры алюминиевого зеркала $\mathrm{Al}(E x p)$ несколько отличаются от табличных, возможно вследствие образования оксидной пленки на поверхности металла. 


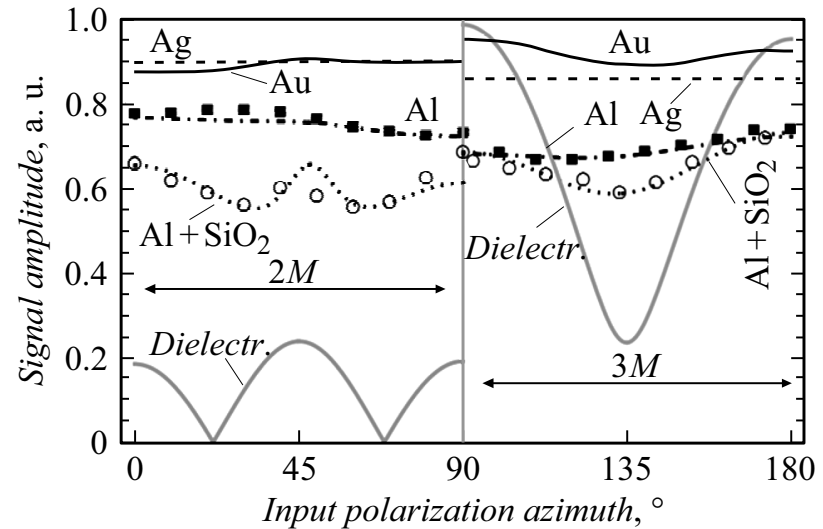

Рис. 3. Зависимость амплитуды сигнала от азимута входной поляризации для схем с двумя $(2 M)$ и тремя (3M) зеркалами из различных материалов. Точки - эксперимент, линии теория.

Для ячейки было принято наиболее общее описание: $C\left(R_{p C}, R_{s C}, \theta_{C}, \delta_{C}\right)=R\left(\theta_{C}\right) D\left(\delta_{C}\right) A\left(R_{p C}, R_{S C}\right)$, позволяющее учесть возможные эффекты линейного дихроизма $\left(R_{p C} \neq R_{S C}\right)$ и линейного двулучепреломления $\left(\delta_{C} \neq 0\right)$, обусловленные выстраиванием магнитных моментов в ячейке. В атомарной системе не может существовать постоянных, т.е. непрецессирующих, магнитных моментов, направленных под углом к магнитному полю (в том числе и вдоль оси $x)$, и величина $\theta_{C} \sim \theta_{A} \cos (\omega t)$ может отличаться от нуля только на частоте магнитного резонанса $\omega$ и при определенных условиях на ее второй гармонике. Сигнал магнитного резонанса при этом описывается как производная разностного сигнала на БФП по амплитуде $\theta_{A}$.

Нами были исследованы зависимости поляризационных характеристик пробного луча от азимута $\psi$ входной поляризации $\left(s=0^{\circ}, p=90^{\circ}\right)$ для двух наборов зеркал: $\mathrm{Al}$ и $\mathrm{Al}+\mathrm{SiO}_{2}$. Показано, что поляризационные характеристики пробного луча без ячейки хорошо описываются в рамках модели (рис. $2, a$ ). Введение в систему ячейки приводит к незначительному изменению эллиптичности луча: $\delta_{C}=(-2 \pm 2)^{\circ}$.

Было также исследовано влияние вносимой зеркалами эллиптичности пробного луча на амплитуду сигнала магнитного резонанса. Предпринята попытка скомпенсировать это влияние введением в схему одного или двух расположенных непосредственно перед БФП дополнительных зеркал: зеркало $M 3$ отражает пробный луч в направлении оси $y$, выводя его из плоскости $z 0 x$, зеркало $M 4$ отражает луч в направлении $-z$.

Результат измерений для $\mathrm{Al}$ показан на рис. $2, b$. Видно, что поляризационные зависимости амплитуды сигнала, нормированного на интенсивность света на БФП, действительно могут быть частично скомпенсированы введением одного и, в меньшей степени, двух зеркал. При этом без существенной потери точности можно считать, что помимо поворота угла поляри- зации на ларморовской частоте ячейка не вносит в систему никаких возмущений $\left(\delta_{C}=0, R_{p C}=R_{S C}=1\right)$. Сравнение теории и эксперимента позволяет говорить о достаточной степени апробации теории.

На рис. 3 представлены теоретические и экспериментальные зависимости амплитуды сигнала от азимута входной поляризации для перечисленных выше типов зеркал. Видно, что в стандартной схеме с двумя зеркалами $(2 M)$ потеря сигнала происходит в любом случае: при применении металлических зеркал - из-за их относительно низких (особенно для Al) коэффициентов отражений, а при применении зеркал с защитными покрытиями и диэлектрических интерференционных зеркал из-за вносимой ими фазовой задержки, причем в последнем случае потери сигнала могут быть катастрофичны. Как следует из рис. 3 , именно в этом случае введение в схему зеркала $M 3$ оказывается особенно эффективным: вследствие высокой отражательной способности диэлектрических зеркал оно позволяет исключить потери при условии, что поляризация входного излучения представляет собой чистую $s$ - или $p$-поляризацию.

Таким образом, исследовано влияние на амплитуду сигнала магнитного резонанса поляризационных характеристик пробного луча, определяемых потерями и фазовыми задержками, которые вносят элементы оптической схемы. Построена и экспериментально апробирована теория, проведено сравнение влияния диэлектрических и металлических зеркал, предложен способ компенсации этого влияния. Наиболее существенным практическим выводом является рекомендация использовать в стандартной схеме датчика металлические (золотые и серебряные) зеркала без защитных покрытий. Использование же диэлектрических интерференционных зеркал, несмотря на их высокую отражательную способность, оказывается оправданным только при введении в схему дополнительного компенсирующего элемента и только для $s$ - и $p$-поляризаций лазерного излучения.

\section{Благодарности}

Авторы выражают благодарность В.С. Запасскому за интерес к работе и полезные дискуссии.

\section{Конфликт интересов}

Авторы заявляют, что у них нет конфликта интересов.

\section{Список литературы}

[1] Budker D., Romalis M. // Nature Phys. 2007. V. 3. P. 227.

[2] Александров Е.Б., Вершовский А.К. // УФН. 2009. Т. 179. № 6. C. 605-637.

[3] Serkland D.K., Geib K.M., Peake G.M., Lutwak R., Rashed A., Varghese M., Tepolt G., Prouty M. // Proc. SPIE. 2007. V. 6484. P. 648406 (1-10).

[4] Малеев Н.А., Блохин С.А., Бобров М.В., Кузьменков А.Г., Кулагина, М.М., Устинов В.М. // Гироскопия и навигация. 2018. T. 26. № 1. C. 81-92. 
[5] Shah V.K., Wakai R.T. // Phys. Med. Biol. 2013. V. 58. N 22. P. 8153-8161.

[6] Korth H., Strohbehn K., Tejada F., Andreou A.G., Kitching J., Knappe S., Lehtonen S.J., London S.M., Kafel M. // J. Geophys. Res. Space Phys. 2016. V. 121. N 8. P. 7870-7880.

[7] Walker T.G., Larsen M.S. // Advances in atomic, molecular, and optical physics / Eds E. Arimondo, C.C. Lin, S.F. Yelin. Academic Press, 2016. V. 65. P. 373-401.

[8] Вершовский А.К., Литманович Ю.А., Пазгалёв А.С., Пешехонов В.Г. // Гироскопия и навигация. 2018. Т. 26. № 1. C. 55-80.

[9] Bhaskar N.D., Camparo J., Happer W., Sharma A. // Phys. Rev. A. 1981. V. 23. N 6. P. 3048-3064.

[10] Kominis I.K., Kornack T.W., Allred J.C., Romalis M.V. // Nature. 2003. V. 422. N 6932. P. 596-599.

[11] Ledbetter M.P., Savukov I.M., Acosta V.M., Budker D., Romalis M.V. // Phys. Rev. A. 2008. V. 77. N 3. P. 033408.

[12] Boto E., Holmes N., Leggett J., Roberts G., Shah V., Meyer S.S., Muñoz L.D., Mullinger K.J., Tierney T.M., Bestmann S., Barnes G.R., Bowtell R., Brookes M.J. // Nature. 2018. V. 555. P. 657.

[13] Bell W.E., Bloom A.L. // Phys. Rev. 1957. V. 107. N 6. P. $1559-1565$.

[14] Smith L.L., Koch P.M. // J. Opt. Soc. Am. A. 1996. V. 13. N 10. P. 2102-2105.

[15] Galvez E.J., Koch P.M. // J. Opt. Soc. Am. A. 1997. V. 14. N 12. P. 3410-3414.

[16] Вершовский А.К., Пазгалев А.С. // ЖТФ. 2008. Т. 78. В. 5. C. $116-124$

[17] McMaster W.H. // Am. J. Phys. 1954. V. 22. N 6. P. 351-362.

[18] McMaster W.H. // Rev. Mod. Phys. 1961. V. 33. N 1. P. 8-28.

[19] Born M., Wolf E. Principles of optics. 7th ed. Cambridge: Cambridge University Press, 1999. P. 621. 